\title{
Characterization of Zinc Soap from an Accelerated Aged Oil Painting Test Panel
}

\author{
Thomas Lam, Christine Romano, G. Asher Newsome, Nicole Little, and Jia-Sun Tsang
}

Museum Conservation Institute, Smithsonian Institution, Suitland, MD, USA

Zinc white is a commonly used oil paint and ground found in modern and contemporary paintings. Crystalline and amorphous zinc soaps are formed by interactions of zinc oxide pigments and free fatty acids in oil paint and are known to cause paint deterioration in the form of brittleness, efflorescence, delamination, and protrusions. This study aims to gain a better understanding of protrusions from an oil paint test panel using correlative spectroscopies. A test panel was designed to mimic the structure of a layered oil painting using known materials, with a lead white oil ground over which zinc white and cadmium red deep oil paints were layered. As the formation of zinc soaps can take several years, the test panel was accelerated aged [1]. A temperature and humidity benchtop unit from ESPEC was used to cycle between $15{ }^{\circ} \mathrm{C}$ and $20 \%$ relative humidity $(\mathrm{RH})$ and $50{ }^{\circ} \mathrm{C}$ and $80 \% \mathrm{RH}$ for 10 hours with a 2 hour ramp between set-points for a total of 672 hours. Within another four years of natural aging, metal soap protrusions were observed. Protrusions were sampled, embedded in Bio-Plastic ${ }^{\circledR}$ (synthetic polyester resin), and dry polished. $\mu$-Fourier transform infrared (FTIR) non-contact mapping in reflectance mode was carried out with a Thermo Nicolet 6700 FTIR spectrometer equipped with a mercury cadmium telluride type A detector, in addition to attenuated total reflection (ATR)-FTIR. Uncoated samples were analyzed in variable pressure mode using a Hitachi S3700-N scanning electron microscope (SEM). Electron dispersive spectroscopy (EDS) was performed using a Bruker XFlash 4010 detector with Esprit software. Samples were analyzed at $15 \mathrm{kV}$ accelerating voltage. X-ray diffraction (XRD) analysis was performed using a Rigaku D/Max Rapid Micro X-ray with $\mathrm{Cu}$ Ka radiation at 50 kV, 40 mA and 2 kW.

From a polished aerial view, white opaque regions and a gel-like feature were found within the protrusion (Fig. 1A). The composite EDS map of the selected elements of $\mathrm{Cd}$ (red), $\mathrm{Zn}$ (green), $\mathrm{C}$ (blue), O (white) showed the gel-like feature to be lower in O concentration (dark blue in Fig. 1B) than the Bio-Plastic ${ }^{\circledR}$ (lighter blue in Fig. 1B), which was confirmed by extracted background normalized EDS spectra (Fig. 1C) from the boxed areas shown in (Fig. 1B). Reference spectra of zinc stearate, a common soap, were collected in both ATR and reflectance mode. Zinc stearate has absorbance peaks at $1533 \mathrm{~cm}^{-1}, 1456 \mathrm{~cm}^{-1}$, and $1396 \mathrm{~cm}^{-1}$ wavenumbers (shown in Fig. 2A in ATR-FTIR). However, the position of the peaks did shift slightly, and scaling intensity was not preserved in FTIR reflectance mode (Fig. 2B). ATR-FTIR of the gel-like feature showed the three absorbance peaks associated with zinc stearate along with a stretching $\mathrm{C}=\mathrm{O}$ at $1732 \mathrm{~cm}^{-1}$, which is likely a product from the linseed oil (Fig. 2C). The extracted FTIR spectra from the mapped gel-like region also showed three weaker and shifted absorbance peaks that are associated with zinc stearate in reflectance mode (Fig. 2D).

XRD was performed on a protrusion mounted on a glass fiber with PVAC-based synthetic glue. The gellike region was found to be amorphous, with surrounding zincite $(\mathrm{ZnO})$ particles from the paint. Gas chromatography-mass spectrometry of micro-sampled protrusions will be performed with an Agilent 6890-5975 GCMS to gain further insights into the organic composition of the soaps.

References:

[1] D. Erhardt et al. Studies in Conservation, 50 (2005), p. 143-150. 


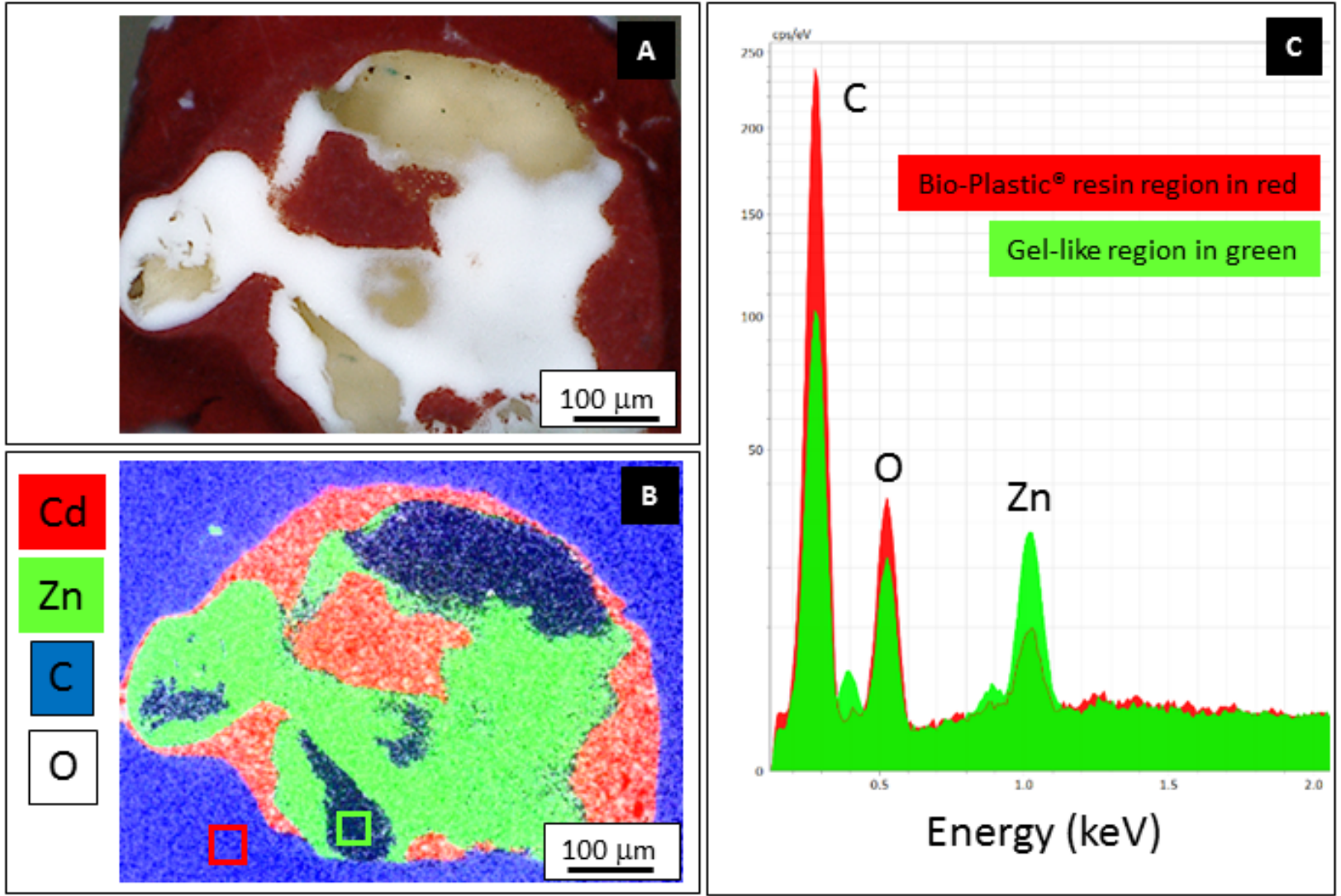

Fig. 1. A) Optical micrograph of an embedded and polished $\mathrm{Zn}$ protrusion in aerial view. B) Composite SEM-EDS map (Cd-red, Zn-green, C-blue, O-white) with C) EDS spectra extracted from boxed regions of bioplastic (red) and gel-like feature (green) in the low keV energy range.
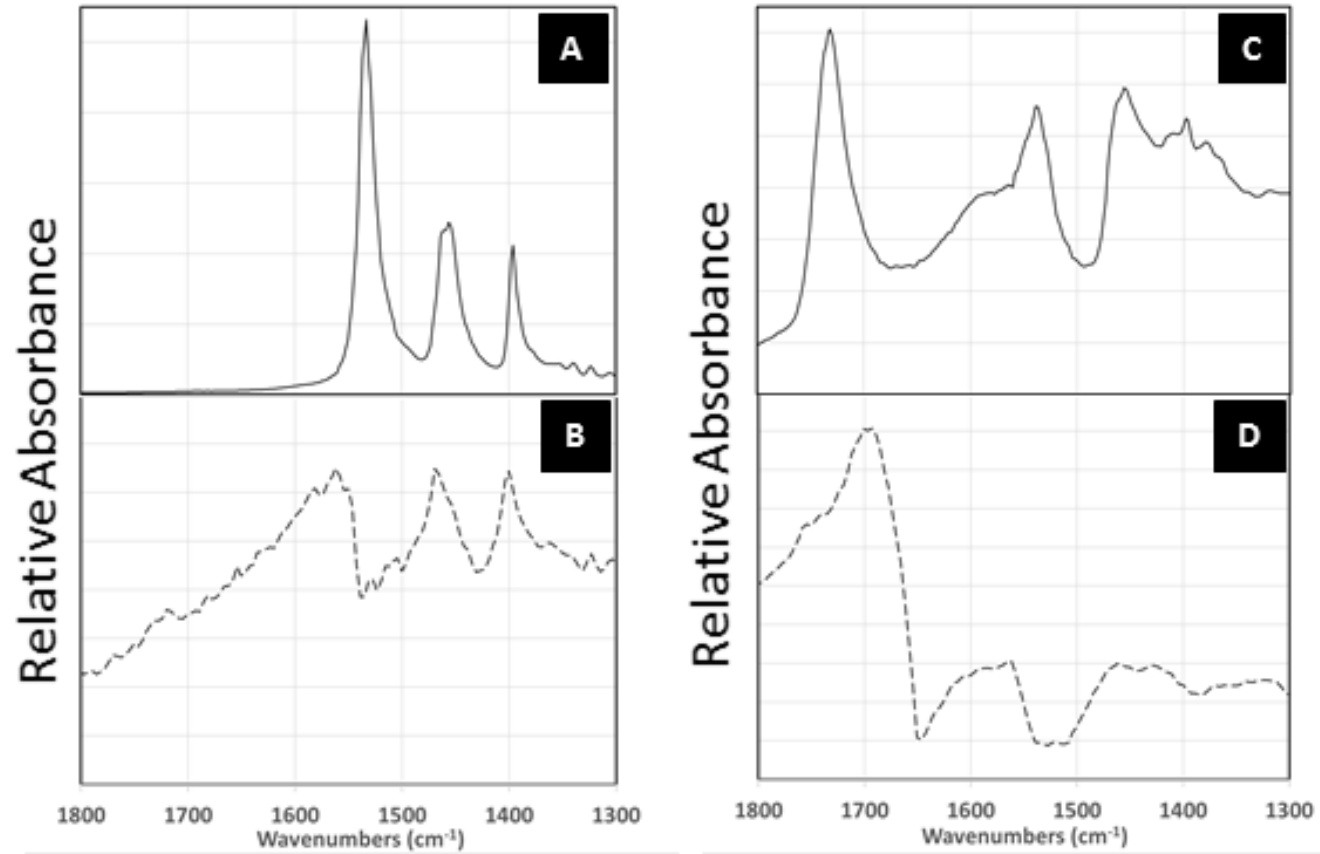

Fig. 2. A) ATR-FTIR reference spectra for zinc stearate. B) Reflectance FTIR reference spectra for zinc stearate. C) ATR-FTIR spectra for gel-like substance from a protrusion. D) Reflectance FTIR spectra of gel-like region of the protrusion in the approximate location of the green box in Fig 1B. 